\title{
Thyrocervical trunk-external carotid artery bypass for positional cerebral ischemia due to common carotid artery occlusion
}

\author{
Report of three cases
}

\author{
Miguel A. Melgar, M.D., Ph.D., And Martin E. Weinand, M.D. \\ Division of Neurosurgery, University of Arizona College of Medicine, Tucson, Arizona
}

\begin{abstract}
Medically refractory positional cerebral ischemia and concomitant orthostatic hypotension associated with chronic common carotid artery (CCA) occlusion are rare. The authors detail their experience with three cases treated exclusively by an extracranial bypass in which the thyrocervical trunk was used as the donor vessel. Postoperatively grafts were patent and symptoms resolved in all three patients, although orthostatic hypotension remained. Postural cerebral ischemia due to CCA occlusion can be treated by extracranial bypass surgery. The thyrocervical trunk is a suitable donor for reconstruction of the external carotid artery in these cases.
\end{abstract}

\section{KEY WORDS • thyrocervical • carotid occlusion • bypass surgery • positional cerebral ischemia}

Positional cerebral ischemia triggered by standing, with or without orthostatic hypotension, is thought to be caused by hemodynamic cerebrovascular insufficiency associated with stenosis or occlusion of a proximal major extra- or intracranial artery. ${ }^{6,916}$ It has been described in cases of basilar artery ${ }^{6}$ and ICA occlusion; ${ }^{6,9,16}$ however, PCI and coexistent orthostatic hypotension associated with CCA occlusion is a rare event. Because of the development of extra/intracranial collaterals most patients with CCA occlusion are asymptomatic. In a few reported series of symptomatic CCA occlusion subclavian to ECA saphenous vein interposition grafting has been the treatment of choice. $^{4,10,17,18,27}$

In this report, we review the phenomena of PCI and orthostatic hypotension, and describe a technical variant for ECA reconstruction in CCA occlusion by using the thyrocervical trunk as the donor vessel in three refractory cases of PCI presented to the neurosurgery service between October 1997 and December 2002.

Abbreviations used in this paper: $\mathrm{CA}=$ carotid artery; $\mathrm{CBF}=$ cerebral blood flow; $\mathrm{CCA}=$ common $\mathrm{CA}$; $\mathrm{CT}=$ computerized tomography; ECA = external CA; ICA = internal CA; MCA = middle cerebral artery; $\mathrm{MR}=$ magnetic resonance; $\mathrm{OA}=$ ophthalmic artery; $\mathrm{PCI}=$ positional cerebral ischemia $; \mathrm{PET}=$ positron emission tomography; SPECT = single-photon emission CT; STA = superficial temporal artery; SVG = saphenous vein graft; VA = vertebral artery.

\section{CASE REPORTS}

\section{Case 1}

This 41-year-old man presented to the neurology service with severe episodes of lightheadedness and blurred vision upon standing. These episodes were repetitive $(>$ two per day) and brief ( $<5$ seconds). Medical history included arterial hypertension, and symptoms continued despite significant reduction of his antihypertensive medications. Cardiac, dysautonomic, and neurotological disorders were ruled out. Medical treatment for his disabling symptoms included head-up tilt at night, as well as physical counter maneuvers such as squatting, bending forward during the crisis, and the use of an abdominal binder. Pharmacological therapy included fludrocortisone acetate $(0.1 \mathrm{mg}$ once a day, increased by $0.1-\mathrm{mg}$ increments at 1 -week intervals up to $0.3 \mathrm{mg}$ daily), but side effects such as headaches and hypokalemia were poorly tolerated. He also received ephedrine (30 $\mathrm{mg}$ three times daily) with no response.

Neurological examination demonstrated no abnormality. Supine blood pressure was 140/80 mm Hg (both arms), which changed to $120 / 75 \mathrm{~mm} \mathrm{Hg}$ in both arms when erect. We also noted blurred vision, perioral paresthesias, lightheadedness, and left-handed clumsiness that appeared after standing for 10 seconds and lasted 15 seconds before subsiding. Head CT scanning and MR imaging revealed 
chronic periventricular ischemic lesions. Aortic arch angiography demonstrated bilateral CCA occlusion and welldeveloped thyrocervical trunks (Fig. 1 upper left). The left ECA was entirely reconstituted by retrograde flow from left VA muscular branch-occipital artery anastomoses, and arterial supply from thyrocervical trunk and ascending cervical arteries (Riles Type $1 \mathrm{~B}^{21}$ ) (Fig. 1 upper right). The ascending pharyngeal artery anastomosed with and reconstituted the petrous and cavernous ICA (Fig. 1 upper right). Left VA injection revealed a patent dominant

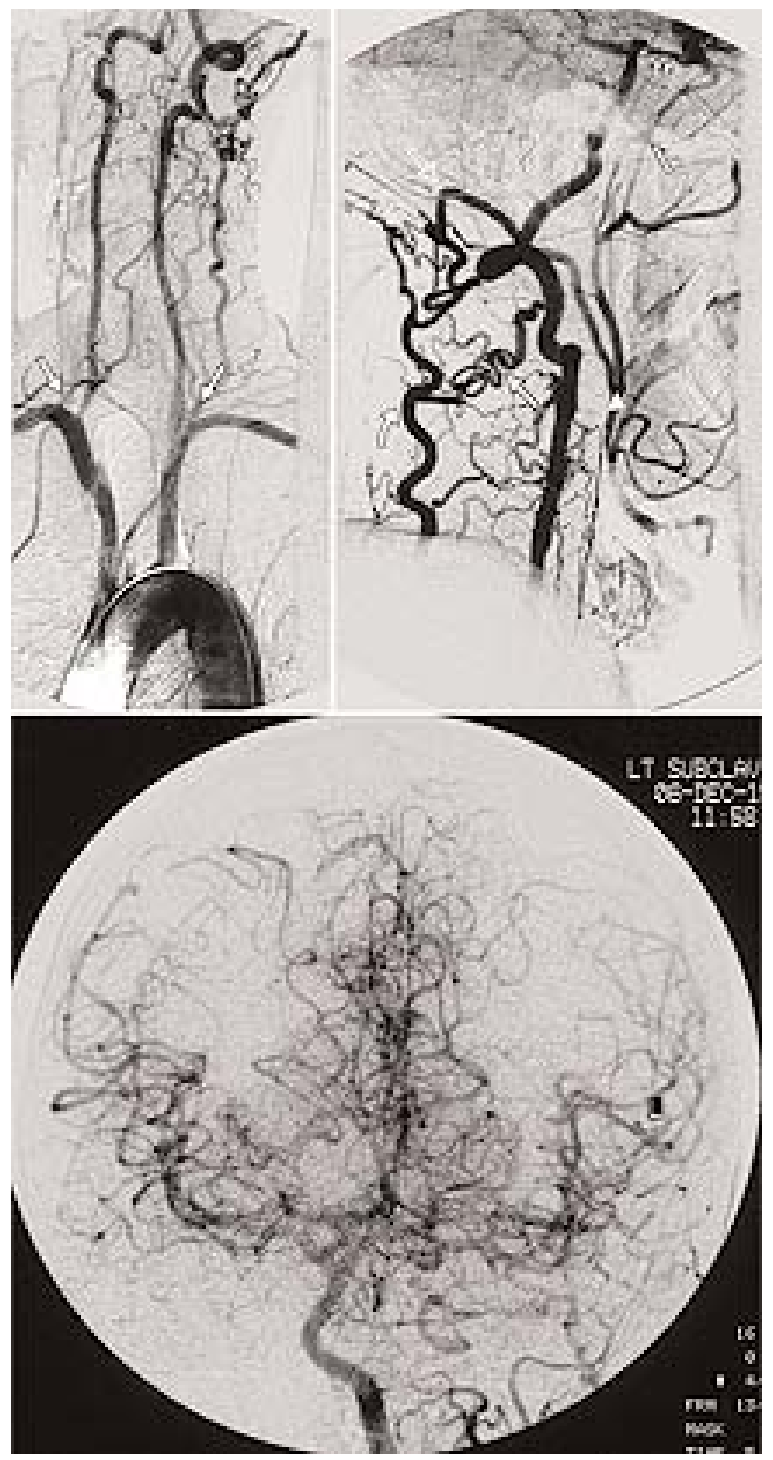

Fig. 1. Preoperative angiograms. Upper Left: Aortic arch injection revealing bilateral CCA occlusion and well-developed thyrocervical trunk arteries (arrows) Upper Right: Left VA injection demonstrating anastomoses between a large vertebral muscular branch (arrow) and ascending cervical artery (curved arrows) and OA (open arrow). The ECA (arrowhead) is entirely reconstituted by retrograde flow (Riles Type 1B). The ascending pharyngeal (half arrow) and posterior communicating arteries (small double arrows) reconstitute the petrous and cavernous segment of the left ICA. Lower: Left VA injection filling both the anterior and posterior circulations. artery filling the vertebrobasilar system and cerebral hemispheres via patent posterior communicating arteries (Fig. 1 lower). The right VA was hypoplastic and ended as a posterior inferior cerebellar artery. Single-photon emission CT revealed baseline bilateral hypoperfusion and poor cerebrovascular reserve after injection of acetazolamide. The patient declined anticoagulant medications. Neurosurgery was consulted and an augmentative CBF procedure was planned.

\section{Case 2}

This 76-year-old man presented to the neurology service with several daily episodes of confusion, left-handed clumsiness, and leg shaking upon standing. These spells subsided within a few minutes. Medical history included atrial fibrillation treated with warfarin. Neurological examination was normal. Systolic blood pressure decreased from supine to upright position by $30 \mathrm{~mm} \mathrm{Hg}$ in both arms (supine 160/85 mm Hg; upright 130/80 mm Hg). The patient presented with left-handed weakness and blurred vision in the left eye that lasted 20 seconds. These symptoms subsided while the patient stood. Syncope, dysautonomic, and inner ear problems were ruled out. It was recommended that his head of the bed be elevated by $20 \mathrm{~cm}$ at night; additionally, the same counter maneuvers as described in Case 1 were recommended. He also received fludrocortisone acetate $(0.3 \mathrm{mg}$ daily) and ephedrine (15 $\mathrm{mg}$ three times daily) but no response occurred. He was particularly sensitive to ephedrine-induced side effects that included tachycardia and tremulousness. Head CT and MR imaging revealed chronic ischemic changes around the periventricular white matter. Aortic arch angiography revealed right CCA occlusion, a patent right thyrocervical trunk, and hypoplastic right VA that was occluded at the C-2 level (Fig. 2 upper left). Right subclavian injection revealed reconstitution of the right ECA and, to a lesser extent, the right ICA from retrograde flow via right VAOA anastomoses (Riles Type 1A) (Fig. 2 upper right). The left VA was dominant and filled the vertebrobasilar system and, to a limited extent, the right MCA territory (Fig. 2 lower left). Leptomeningeal anastomoses between the right posterior cerebral artery and MCA were observed (Fig. 2 lower left). Left CA injection revealed normal patency and filling of both anterior cerebral arteries with no crossover flow to the right (Fig. 2 lower right). Singlephoton emission CT revealed baseline hypoperfusion on the right that worsened after acetazolamide administration. Neurosurgery was consulted and an augmentative $\mathrm{CBF}$ procedure was recommended.

\section{Case 3}

This 67-year-old man was admitted to the neurology service because of repetitive transient episodes of left hemiparesis provoked by standing. These brief episodes occurred two to three times per week. Medical history included peripheral vascular disease and bilateral femoralpopliteal bypass surgery. He tolerated pharmacological therapy with fludrocortisone acetate and ephedrine better than the patients in Cases 1 and 2, but no improvement in symptoms was observed. Warfarin-based anticoagulation therapy was initiated but no response occurred. Neurological examination revealed an awake, alert, and oriented 


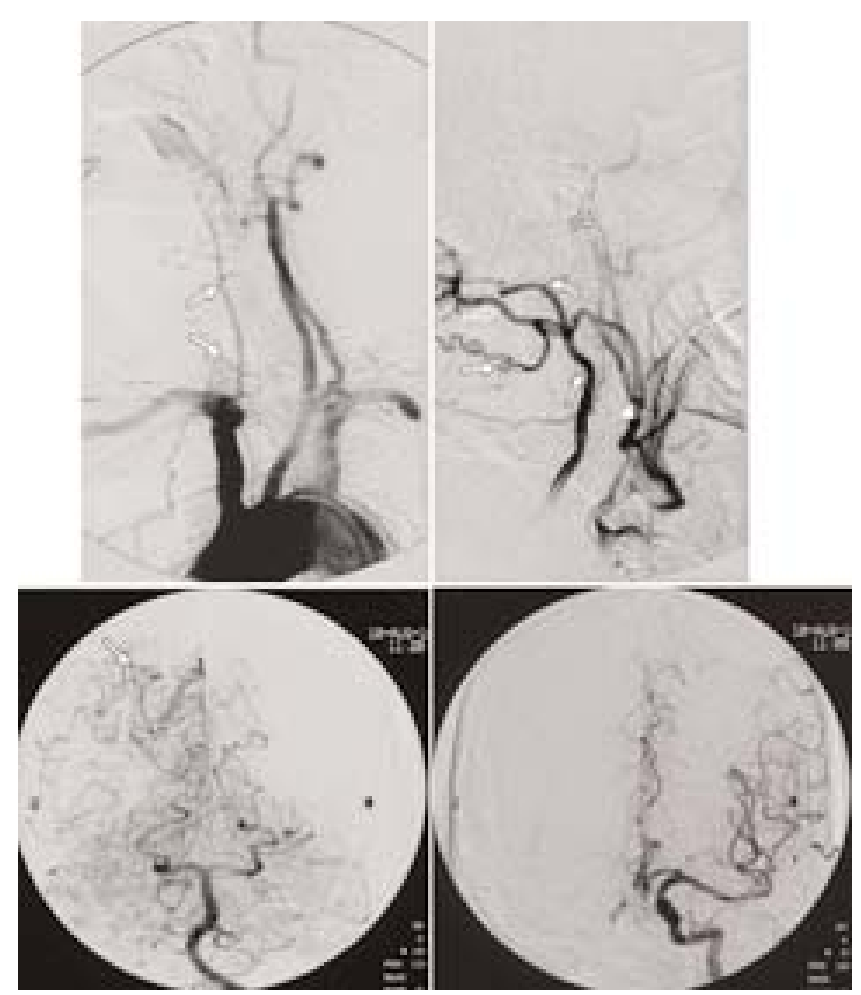

Fig. 2. Case 2. Preoperative angiograms. Upper Left: Aortic arch injection demonstrating right CCA occlusion, hypoplastic right VA (arrow), and patent thyrocervical trunk (curved arrow). Upper Right: Right VA injection revealing occlusion at the C-2 vertebral level (solid arrow). Large muscular branches of the VA (curved solid arrows) reconstitute the OA (open arrow). Retrograde flow from the OA recanalizes the ECA (arrowhead) and ICA (double arrows) (Riles Type 1A). Lower Left: Left VA injection revealing faint filling of the right hemisphere. Leptomeningeal anastomoses between the right PCA and MCA are present (arrow). Lower Right: Left CA injection revealing no crossover flow to the right hemisphere.

man in whom no focal deficits occurred while lying supine. Supine blood pressure was $150 / 80 \mathrm{~mm} \mathrm{Hg}$ (right arm and $155 / 80 \mathrm{~mm} \mathrm{Hg}$ (left arm). One minute after standing, he felt dizzy and mild left hemiparesis (Grade 4/5) and involuntary left lower leg shaking lasting 10 seconds occurred; these symptoms resolved after lying down. Blood pressure while upright was 120/75 $\mathrm{mm} \mathrm{Hg}$ (right arm) and $125 / 75 \mathrm{~mm} \mathrm{Hg}$ (left arm). Cardiac workup revealed no abnormalities. Head CT scanning demonstrated an old small watershed stroke in the right frontoparietal region. Single-photon emission CT scans with acetazolamide challenge revealed poor cerebrovascular reactivity in the right cerebral hemisphere. Aortic arch angiography demonstrated right CCA occlusion, hypoplastic right VA, and a patent right thyrocervical trunk (Fig. 3 upper left). Right subclavian injection revealed VA occlusion and anastomosis between VA muscular branches and OA with retrograde filling of the right ECA (Riles Type 1B) (Fig. 3 upper right). The left VA was dominant, filling the entire vertebrobasilar system. Left CCA angiography revealed normal findings, with filling of the right cerebral hemisphere via left-to-right crossover flow by a patent anterior communicating artery (Fig. 3 lower left). Three months later symptoms persisted and the patient underwent neurosurgical consultation. Brain computerized tomography scans and MR imaging demonstrated no new lesions. We planned surgical treatment for this patient.

\section{Description of Surgical Intervention}

A staged treatment plan was aimed at augmenting ECA blood flow in the patients in Cases 1 and 3. The goal was to engorge a patent STA for an STA-MCA bypass to be performed in the immediate future. Additionally, the possibility that the first-stage procedure may suffice for correcting $\mathrm{CBF}$ deficits was considered. The goal in the patient in Case 2 was to obtain direct CBF improvement by increasing flow in the partially reconstituted right ICA.

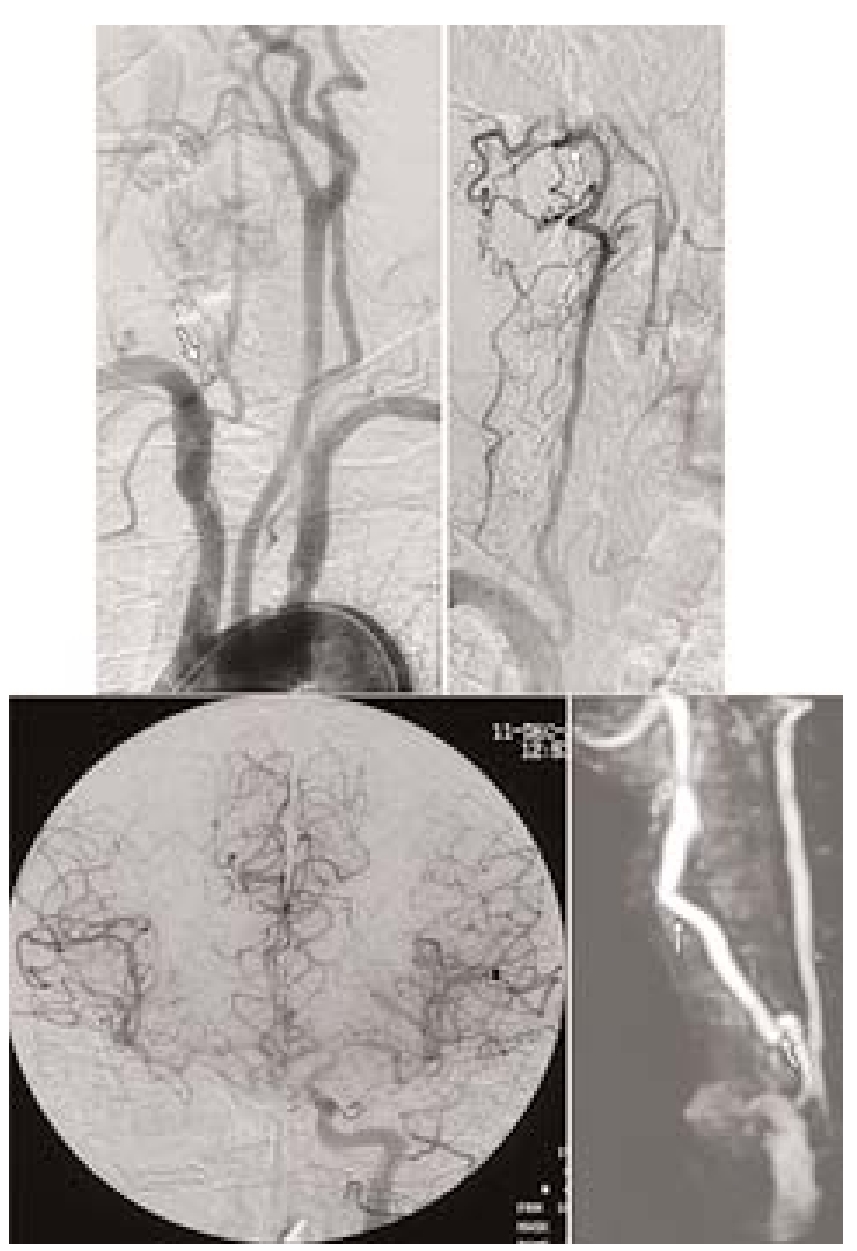

Fig. 3. Case 3. Preoperative angiograms and postoperative MR angiogram. Upper Left: Aortic arch injection revealing right CCA occlusion and patent thyrocervical trunk (arrow). Upper Right: Right subclavian injection demonstrating VA occlusion at the skull base (arrow). Muscular branches off the VA reconstitute the occipital artery (curved arrow), which by retrograde flow fills the ECA (curved open arrows). Lower Left: Left ICA injection demonstrating good crossover flow to the right hemisphere. Lower Right: Postoperative MR angiogram demonstrating patent saphenous vein graft (arrow) interposed between the thyrocervical trunk (curved arrow) and ECA (curved open arrow). 
We chose to anastomose the thyrocervical trunk to the patent ECA. In brief, the patient was placed supine and the head turned $45^{\circ}$ away from the affected side. A longitudinal incision was made along the medial border of the sternocleidomastoid muscle. The occluded CCA and the thyrocervical trunk were identified deeper and medial to the internal jugular vein (Fig. 4 upper). Identification of the most medially located VA origin helped to orient the surgeon for the dissection. The patient received an intravenous 5000-U bolus of heparin. A proximal and distal endto-side interposition SVG anastomosis was performed. We used No. 7-0 and No. 6-0 Prolene sutures for the proximal and distal anastomoses, respectively. The graft lay under and medial to the sternocleidomastoid muscle (Fig. 4 lower).

The postoperative courses were uneventful in all three patients. Strong pulse in the STA was noticed. Symptoms subsided immediately in Cases 2 and 3, and progressively improved in Case 1. At 5-year follow-up examination in the patient in Case 1 and 3-year in the patient in Case 2, no PCI spells were reported, although orthostatic hypotension remained. This led us to believe that there was no need for a second-stage STA-MCA bypass procedure in these patients. In the patient in Case 2, acetazolamidebased SPECT performed at 3 months and 1 year postoperatively revealed normal CBF. Despite the absence of symptoms in the patient in Case 1, SPECT demonstrated normal baseline but persistent impaired cerebrovascular

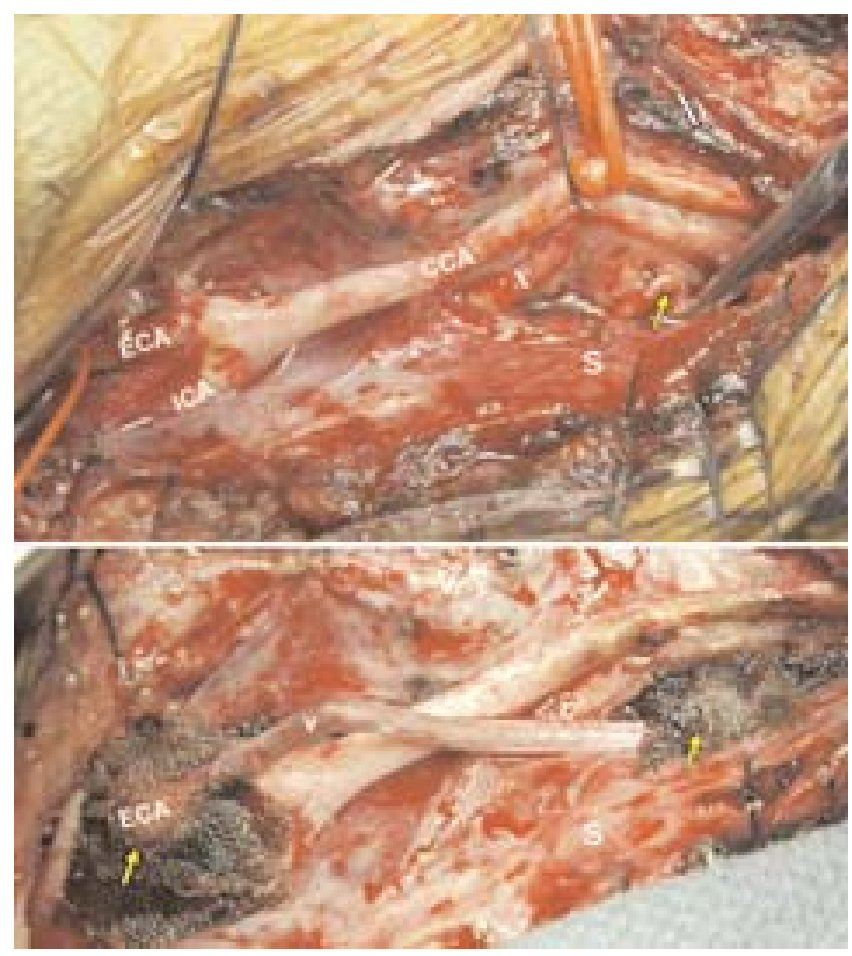

Fig. 4. Case 3. Intraoperative photographs. Upper: The occluded CCA is retracted by a vessel loop. The thyrocervical trunk (arrow) is pointed by a forceps. Notice its medial position in relation to the internal jugular vein, in this case covered by the carotid sheath (S). Lower: Bypass completed. The anastomotic sides (arrows) are covered by hemostatic material. $\mathrm{X}=$ vagus; $\mathrm{V}=$ saphenous vein graft. reactivity to acetazolamide at 3 and 16 months. Of interest, this patient remains free of symptoms despite unchanged postoperative postural decrease in his systolic pressure (20-30 $\mathrm{mm} \mathrm{Hg}$ ).

Postoperative angiography conducted at 3 months and 1 year in Cases 1 and 2 revealed patent anastomoses. Immediate postoperative MR angiography in Cases 3 also demonstrated a patent bypass (Fig. 3 lower and right). In this patient, although asymptomatic, postural hypotension remains. We plan on performing SPECT at 3-month follow-up examination.

\section{DISCUSSION}

\section{Positional Cerebral Ischemia and Postural Hypotension}

Our three patients exhibited characteristic and reproducible symptoms related to positional cerebral ischemia. This phenomenon has been previously described in few reports and correlated with angiographically proven main extra- or intracranial cerebral vessel stenosis or occlusion. ${ }^{6,9,16}$ The prevalence of asymptomatic ${ }^{8}$ and symptomatic $^{11,17,18}$ main extracranial-intracranial vessel occlusion, however, is higher than reproducible PCI encountered in clinical practice. The reasons for this remain unknown, although two explanations may be plausible: 1) the angiographic pattern of collateral circulation ${ }^{14,19,25}$ and 2) impaired function of baroreceptors afferents at the carotid sinus. ${ }^{1}$ Attempts have been made to correlate the angiographic pattern of collateral circulation in chronic CA occlusion with CBF status. ${ }^{14,19,25}$ Thus, a Willisian-type collateral or crossover flow through a patent anterior communicating artery has shown less impaired cerebrovascular reactivity than OA retrograde flow, and much less than leptomeningeal-type collateral pattern. ${ }^{14,25}$ The latter has been associated with a state of maximal hemodynamic compromise on Xe-CT $\mathrm{CBF}^{25}$ and PET studies. ${ }^{20}$

In the seminal study reported by Powers, et al.,${ }^{20}$ the authors used PET to demonstrate that patients with chronic CA occlusion and abnormal oxygen extraction fraction and steal phenomenon, referred to as Stage II hemodynamics, depend mainly on leptomeningeal collateral vessels for maintenance of CBF ipsilateral to the affected side. Furthermore, the same group has shown an increased risk for stroke in this population. ${ }^{11}$ We speculate that PCI may have been a harbinger of impending stroke in the patient in Case 2 because he depended on leptomeningeal collateral blood flow. Because acetazolamide-based SPECT is a qualitative test that reflects no absolute CBF values and relies on comparative flow between two hemispheres, its reliability has been questioned. ${ }^{26}$ Some authors, however, have shown that it may detect cases of Stage II hemodynamic failure, proven by PET, performed in the same setting. ${ }^{12}$ Additionally, acetazolamide-based SPECT has identified patients with CBF dependent on leptomeningeal collateral flow. ${ }^{19}$

Although poor cerebrovascular reserve was demonstrated by SPECT in the patients in Cases 1 and 3, their respective angiographically demonstrated collateral patterns were not leptomeningeal dependent, and thus a different explanation for their symptoms may be elaborated. Akinola, et al., ${ }^{1}$ have shown that in patients with chronic uni- or bilateral severe CA stenosis, the incidence of pos- 
tural hypotension is higher than in hypertensive patients and normotensive controls. In the same study, postural hypotension was not related to sympathetic efferent failure, as demonstrated by normal catecholamine production in response to standing. Rather, impaired baroreceptor afferents in the region of the carotid sinus were thought to be the cause. ${ }^{1}$ Whether this neural-mediated mechanism accounted for the symptoms in our patients is unknown. Although we did not measure catecholamine levels, the lack of response to ephedrine, a noradrenaline-releasing drug, ${ }^{1}$ may suggest that sympathetic failure was not the underlying mechanism for hypotension in the present report.

It is possible that postural hypotension exhibited in Cases 1 and 3 triggered a reduction on CBF despite the good collateral pattern demonstrated on angiography. Medical management of orthostatic hypotension caused by diabetes, and treatment for ischemic heart disease and hypertension, improved the rate of transient ischemic attacks in a population with hemodynamically significant ICA stenosis or arteriography-demonstrated occlusion. ${ }^{9}$ Despite reduction of antihypertensive medications, the patient in Case 1 continued to experience transient ischemic attacks that subsided after surgery. Nonetheless, it is difficult to reconcile his neurological improvement with persistent postoperative postural arterial hypotension and $\mathrm{CBF}$ impairment demonstrated on SPECT. Because SPECT relies on crosscomparison between the two cerebral hemispheres under the assumption that one harbors normal baseline $\mathrm{CBF}$, its reliability in cases of bilateral $\mathrm{CCA}$ occlusion may be marginal. In the patient in Case 1, it is possible that PET scanning could have revealed his actual cerebral hemodynamic status, presumably improved by surgery.

\section{Bypass Procedures for Common CCA Occlusion}

Common CA occlusion has been found in approximately 4 to $5 \%$ of patients undergoing cerebral angiography evaluation for cerebrovascular symptoms. ${ }^{7,22} \mathrm{Al}-$ though the natural history of asymptomatic CCA occlusion appears to be benign, ${ }^{8}$ in symptomatic patients it portends a poor prognosis. ${ }^{2,18}$ Riles, et al., ${ }^{21}$ classified CCA occlusion into four types. Type 1 of CCA occlusion involves a patent ICA and/or ECA. Type 1 includes three subtypes including: Type 1A, a patent ICA, and ECA; Type 1B, a patent ECA and occluded ICA; Type 1C, patent ICA and occluded ECA. Type 2 involves complete CCA, ICA and ECA occlusion. In series involving vascular surgery, most cases of symptomatic CCA occlusion in which extracranial/extrathoracic reconstructive bypass procedures have been performed as the definitive treatment are Type 1A and 1B CCA occlusion. ${ }^{18,21}$ When occlusion of both the CCA and ICA is present (Riles Type 1B), bypass procedures to ECA with or without endarterectomy have been shown to improve symptoms in most patients. ${ }^{4,10,22,23,27}$ In the neurosurgical literature, however, CCA occlusion and ECA reconstructive bypass procedures have been reported only as the first stage for an STA-MCA bypass performed at the same or separate session. ${ }^{3,7,15}$ Of interest, despite the disparity in surgical treatment, the authors of both vascu$\operatorname{lar}^{4,10,17,21,22,23,27}$ and neurosurgical series ${ }^{7,15}$ have reported good outcomes in symptomatic patients with CCA occlu- sion. Because ECA may provide as much as $30 \%$ of ipsilateral $\mathrm{CBF}$ in CCA occlusion, ${ }^{18,27}$ it is possible that simple ECA reconstruction may have been sufficient therapy in some patients of those neurosurgical series ${ }^{7,15}$ as it was in the present report. Because of the absence of pre- and postoperative quantitative $\mathrm{CBF}$ studies in the majority of patients, it is difficult to determine the magnitude of $\mathrm{CBF}$ changes that correlated to neurological improvement in those cohorts. ${ }^{4,7,10,15,17,21,22,23,27}$

In the present report, a saphenous vein interposition graft was used for reconstruction of the ECA. This is the most common technique performed for CCA occlusion with the subclavian artery as the donor vessel. ${ }^{4,10,18} \mathrm{Al}$ though thyrocervical trunk-VA bypass has been described in cases of proximal VA stenosis, ${ }^{5}$ to our knowledge there have been no reported cases of thyrocervical trunk-ECA interposition SVG bypass for CCA occlusion. Although two suture lines are also required, we found this technique straightforward and easier to perform than subclavianECA interposition graft bypass. We also found no technical problems associated with vessel mismatch.

The thyrocervical trunk gives rise to three main branches including the inferior thyroid, the superficial or transverse cervical, and the suprascapular arteries. The superficial cervical artery gives rise to the ascending cervical artery. Because all three main branches have approximately the same diameter ${ }^{5,15}$ they are all suitable as donor vessels for an interposition graft. Furthermore, they may enlarge in cases of chronic CCA occlusion (Fig. 1 upper left). Because of its ascending course and cephalad flow direction, however, we believe the inferior thyroid artery is more suitable than the other two branches. The suprascapular artery travels downward deep and below the clavicle superficial to the anterior scalene muscle, and its dissection is more demanding. The superficial cervical artery is situated more lateral in the operative field, although in some cases it may constitute a long pedicle graft that may be mobilized superiorly to reach the ECA. ${ }^{15}$

Ingenious methods of revascularization have been reported for symptomatic CCA occlusion. Kobayashi, et al. ${ }^{15}$ anastomosed the transverse cervical artery to the ECA and then performed an STA-MCA bypass in the same session in two patients with CCA occlusion. Although a low-flow bypass, this procedure was sufficient for reversing acetazolamide-based SPECT-demonstrated $\mathrm{CBF}$ abnormalities in those patients, although no data were available on clinical follow-up examination. ${ }^{15}$ In the present report, we thought that a high-flow bypass was necessary because of the uncertainty of a staged STA-MCA bypass in the immediate future. We speculated that a highflow conduit such as the saphenous vein would make the STA available should extracranial-intracranial bypass be necessary. In low flow bypasses such that provided by the STA, ${ }^{13}$ radial artery, ${ }^{24}$ and transverse cervical artery, ${ }^{15}$ although they may remain patent in the absence of hemodynamic demand, a maturation period is necessary for maximal flow delivery to recipient vascular beds. Thus, in the acute setting they may not be sufficient to override and would rather compete with a preexistent retrograde flow from the OA, and orthograde flow from ascending cervical muscular branches to ECA, as was depicted in Cases 1 and 3. 


\section{CONCLUSIONS}

Symptomatic CCA occlusion, although rare, may present with concomitant positional cerebral ischemia and orthostatic hypotension. Of importance, orthostatic hypotension persists after corrective bypass surgery despite resolution of symptoms. Therefore, collateral blood flow is the determinant factor for the development of postural symptoms in patients with CCA occlusion.

It is imperative to assess carefully the angiographically demonstrated subtype of CCA occlusion for sound surgical planning. In this report, symptoms of positional cerebral ischemia were corrected by thyrocervical-ECA interposition SVG bypass in all three cases. When present, the thyrocervical trunk is a suitable donor in this particular subset of patients.

\section{References}

1. Akinola A, Mathias CJ, Mansfield A, et al: Cardiovascular, autonomic, and plasma catecholamine responses in unilateral and bilateral carotid artery stenosis. J Neurol Neurosurg Psychiatry 67:428-432, 1999

2. Archie JP Jr: Axillary-to-carotid artery bypass grafting for symptomatic severe common carotid artery occlusive disease. J Vasc Surg 30:1106-1112, 1999

3. Ausman JI, Lindsay W, Ramsay RC, et al: Ipsilateral subclavian to external carotid and STA-MCA bypasses for retinal ischemia. Surg Neurol 9:5-8, 1978

4. Berguer R, Bauer RB: Subclavian artery to external carotid artery bypass graft. Improvement of cerebral blood supply. Arch Surg 111:893-896, 1976

5. Brisman MH, Tuhrim S, Jenkins A III, et al: Thyrocervical to vertebral artery transposition and ipsilateral carotid endarterectomy. Surg Neurol 51:327-331, 1999

6. Caplan L, Sergay S: Positional cerebral ischaemia. J Neurol Neurosurg Psychiatry 39:385-391, 1976

7. Collice M, D'Angelo V, Arena O: Surgical treatment of common carotid occlusion. Neurosurgery 12:515-524, 1983

8. Cull DL, Hansen JC, Taylor SM: Internal carotid artery patency following common carotid artery occlusion: management of the asymptomatic patient. Ann Vasc Surg 13:73-76, 1999

9. Dobkin BH: Orthostatic hypotension as a risk factor for symptomatic occlusive cerebrovascular disease. Neurology 39: 30-34, 1989

10. Fry WR, Martin JD, Clagett GP, et al: Extrathoracic carotid reconstruction: the subclavian-carotid artery bypass. J Vasc Surg 15:83-89, 1992

11. Grubb RL Jr, Derdeyn CP, Fritsch SM, et al: Importance of hemodynamic factors in the prognosis of symptomatic carotid occlusion. JAMA 280:1055-1060, 1998

12. Hirano T, Minematsu K, Hasegawa Y, et al: Acetazolamide reactivity on 123I-IMP single photon emission computed tomography in patients with major cerebral artery occlusive disease: correlation with positron emission tomography parameters. J Cerebral Blood Flow Metab 14:763-770, 1994
13. Iwama T, Hashimoto N, Takagi Y, et al: Predictability of extracranial/intracranial bypass function: a retrospective study of patients with occlusive cerebrovascular disease. Neurosurgery 40:53-60, 1997

14. Kluytmans M, van der Grond J, van Everdingen KJ, et al: Cerebral hemodynamics in relation to patterns of collateral flow. Stroke 30:1432-1439, 1999

15. Kobayashi T, Houkin K, Ito F, et al: Transverse cervical artery bypass pedicle for treatment of common carotid artery occlusion: new adjunct for revascularization of the internal carotid domain. Neurosurgery 45:299-302, 1999

16. Marti-Fabregas J, Cocho D, Lleo A, et al: Transcranial Doppler recording in a patient with transient positional cerebral ischemia. Neurology 55:731-732, 2000

17. Martin RS III, Edwards WH, Mulherin JL Jr, et al: Surgical treatment of common carotid artery occlusion. Am J Surg 165: 302-306, 1993

18. McGuiness CL, Short DH, Kerstein MD: Subclavian-external carotid bypass for symptomatic severe cerebral ischemia from common and internal carotid artery occlusion. Am J Surg 155: 546-550, 1988

19. Ozgur HT, Walsh TK, Masaryk A, et al: Correlation of cerebrovascular reserve as measured by acetazolamide-challenged SPECT with angiographic flow patterns and intra- or extracranial arterial stenosis. AJNR 22:928-936, 2001

20. Powers WJ, Tempel LW, Grubb RL Jr: Influence of cerebral hemodynamics on stroke risk: One-year follow-up of 30 medically treated patients. Ann Neurol 25:325-330, 1989

21. Riles TS, Imparato AM, Posner MP, et al: Common carotid occlusion. Assessment of the distal vessels. Ann Surg 199: 363-366, 1984

22. Salam TA, Smith RB III, Lumsden AB: Extrathoracic bypass procedures for proximal common carotid artery lesions. Am J Surg 166:163-167, 1993

23. Schuler JJ, Flanigan DP, DeBord JR, et al: The treatment of cerebral ischemia by external carotid artery revascularization. Arch Surg 118:567-572, 1983

24. Sekhar LN, Duff JM, Kalavakonda C, et al: Cerebral revascularization using radial artery grafts for the treatment of complex intracranial aneurysms: techniques and outcomes for 17 patients. Neurosurgery 49:646-659, 2001

25. Smith HA, Thompson-Dobkin J, Yonas H, et al: Correlation of xenon-enhanced computed tomography-defined cerebral blood flow reactivity and collateral flow patterns. Stroke 25: 1784-1787, 1994

26. Yonas H, Pindzola RR, Meltzer CC, et al: Qualitative versus quantitative assessment of cerebrovascular reserves. Neurosurgery 42:1005-1012, 1998

27. Zarins CK, DelBeccaro EJ, Johns L, et al: Increased cerebral blood flow after external carotid artery revascularization. Surgery 89:730-734, 1981

Manuscript received January 14, 2003.

Accepted in final form February 5, 2003.

Address reprint requests to: Miguel A. Melgar, M.D., Ph.D., Division of Neurosurgery, University of Arizona Medical Center, 1501 North Campbell Avenue, Room 4310, PO Box 245070, Tucson, Arizona 85724-5070. email: melgar@u.arizona.edu. 